\title{
Study Carbon Quantum Dots in Micellar Media
}

\section{Bipin Rooj and Ujjwal Mandal*}

Department of Chemistry, The University of Burdwan, India

*Corresponding author: Ujjwal Mandal, Department of Chemistry, The University of Burdwan, Golapbag, Burdwan, India, 713104, Tel: +918926450862; E-mail: mmujjwal@gmail.com

\section{Conceptual Paper}

Volume 3 Issue 1

Received Date: February 25, 2019

Published Date: March 18, 2019

DOI: $10.23880 /$ macij- 16000135

\section{Abstract}

Carbon quantum dots (CQDs) are believed to play a key role in the field of biomedicine, sensing of biomolecules, bio imaging, targeted drug delivery, energy transfer, etc. CQDs have been successfully employed to detect various metal ions, and important bio-molecules at very low concentration. To explore the potential application of CQDs in bio system it is important to study their property in such systems. The interactions between CQDs and membrane are important in understanding the mechanism of drug action. Micelles are used as a model cell mimicking system. As compared to various other membrane models such as soluble polymers and liposomes, micellar systems are considered to be more advantageous, because of their relative simplicity, low toxicity, and narrow size distribution, long residence time in the system. Hence it is very important to study CQDs in Micellar medium.

Keywords: Liposomes; Micellar Medium; Biomolecules; Bio Imaging; Targeted Drug Delivery; Energy Transfer

Abbreviations: CQDs: Carbon Quantum Dots, PEG: Poly Ethylene Glycol, PL: Photoluminescence.

\section{Introduction}

Carbon quantum dots (CQDs) were accidentally discovered in 2004, after separation and purification of single walled carbon nanotubes synthesized by arcdischarge methods [1]. Since then, the simplicity of synthesis and unique properties of CQDs has attracted lots of interest which are reflected in publications relating their synthetic methodologies optical properties and various applications [2-10]. Carbon quantum dots (CQDs) have inspired extensive studies on them due to their great potential in the biological field and ability to interact with surfactants [11] model lipid bilayers [12], and biomembranes [13].
The physicochemical properties of CQDs, including their fluorescence properties without photo bleaching and photo blinking, their facile emission tuning, excellent chemical and photochemical stability, non-toxicity, reveals their high application potential in vivo biological field [14]. It is believed that CQDs will play an increasing role in analytical and bio analytical science in the near future. The interactions between CQDs and membrane are important in understanding the mechanism of drug action and also provide a detailed inside into more complex biological processes such as the passage of drugs through cell membranes. Owing to the much complex structure of bio-membrane, the less anfractuous models, surfactants micelle having spherical structure, have been used to mimic the bio-membrane environments [15]. As compared to various other membrane models such as soluble polymers and liposomes, micellar systems are considered to be more advantageous, because of their relative simplicity, low toxicity, and narrow size 


\section{Medicinal \& Analytical Chemistry International Journal}

distribution, long residence time in the system, and the enhanced bio-availability and the stability of the drug through the micellar incorporation [16].

The amphiphilic nature of the micelles allows one to study the affinity of small molecules to the biological membranes and hence the physicochemical interactions of CQDs with surfactant can be visualized as an approximation for CQD-membrane interactions. Owing to the pre-eminent importance of interactions of CQDs with surfactant, significant progress can be achieved for better understanding biological interaction of CQDs in exploring their physicochemical aspects in surfactant medium.

Sun and co-workers prepared CDs through laser ablation of a carbon target.3 On the other hand, the "bottom-up" approaches synthesise CQDs from molecular precursors such as citrate and polymer-silica nanocomposites4 through combustion/ thermal treatments, and supported synthetic and microwave synthetic routes. For instance, Liu's team reported a synthetic method based on the use of modified silica spheres as carriers and resols as carbon precursors [4]. Zhu and co-workers showed that CQDs are readily formed by heating a solution of poly (ethylene glycol) (PEG) and saccharide in a $500 \mathrm{~W}$ microwave oven for 2 to $10 \mathrm{~min}$ [5]. Li's team was one of the first groups to introduce the concept of preparing fluorescent CQDs using a simple and green route [6]. Recently Nandi et al. investigated the origin of multi colour fluorescence in carbon dot [7]. Jana and co-workers synthesized fluorescent carbon nanoparticles with a diameter of 2-6 nm from nitric acid oxidation of soot particles and found that the emission quantum yield is size-dependent, that is, the smaller the size, the better is their photoluminescence (PL) efficiency.2 CDs are made up of aggregated 2-pyridone derivatives employing $\pi-\pi$ stacking and H-bonding [8]. The PL emission maximum of $\mathrm{CDs}$ is excitation wavelength independent. Discrete multiple electronic states are involved for the excitation dependent emission in carbon nanodots [9].

The interaction between properly functionalized CQDs with various surfactants like cationic (CTAC), non-ionic (TritonX-100, Brijj-35), zwitter ionic (HPS) and anionic (SDS) surfactants will be revealed using electronic absorption spectroscopy. These results will give an idea about their relative rate of penetration into the micellar system. The titration method of the properly functionalized CQDs as a function of surfactant concentrations will help in evaluating the binding constants. The fluorescence property of CQDs will change with the variation of its local environment and hence can be used to explore the drug-surfactant interactions. Different spectroscopic techniques such as steady state and time resolved fluorescence spectroscopy and ProtonNMR will help to gain some basic information for understanding the interaction between surfactants and CQDs. The fluorescence studies can help us to understand whether the interaction is hydrophobic or electrostatic in nature.

The detail investigation on the nature of interactions between functionalized CQDs and surfactants can be done by fluorescence quenching experiments. An enhancement in the absorbance and blue or red shift with the decrease in fluorescence intensity may be an issue in this purpose. In order to further confirm the results, the number of binding sites in a CQD, binding constants, Stern-Volmer quenching constants, will be evaluated from these spectroscopic techniques.

Different additive effects may be investigated by all of the above spectroscopic techniques. Steady state spectroscopy can investigate the influence of pharmaceutically important co-solvents such as ethanol, ethylene glycol, propylene glycol on the interaction of functionalized CQDS and different surfactants in the concentration range varying from pre micellar to post micellar concentrations region. Questions that may be addressed with this study are:

- What are the factors those control the rate of penetration of CQDs into the core of micellar system.

- The binding constant of different functionalized CQDs and different surfactants will be determined. It will report the affinity of the CQDs towards the organized systems.

- It will also be observed that whether different functionalities of CQDs impart any effect on relative penetration to the core of the normal and reverse micelle. The change of functionalities will also throw some light about the origin of fluorescence properties of the CQDs systems.

- In the presence of surfactants (mimic to bio membrane), these studies will help in designing better and more efficient drug carrier in future.

\section{References}

1. Xu X, Ray R, Gu Y, Ploehn HJ, Gearheart L, et al. (2004) Electrophoretic Analysis and Purification of Fluorescent Single-Walled Carbon Nanotube Fragments. J Am Chem Soc 126(40): 12736-12737. 


\section{Medicinal \& Analytical Chemistry International Journal}

2. Ray SC, Saha A, Jana NR, Sarkar R (2009) Fluorescent Carbon Nanoparticles: Synthesis, Characterization, and Bioimaging Application. J Phys Chem C 113(43): 18546-18551.

3. Sun YP, Zhou B, Lin Y, Wang W, Fernando KAS, et al. (2006) Quantum-Sized Carbon Dots for Bright and Colorful Photoluminescence. J Am Chem Soc 128(24): 7756-7757.

4. Liu RL, Wu DQ, Liu SH, Koynov K, Knoll W, et al. (2009) An Aqueous Route to Multicolor Photoluminescent Carbon Dots Using Silica Spheres as Carriers. Chem Int Ed 48(25): 4598-4601.

5. Zhu H, Wang XL, Li YL, Wang ZJ, Yang F, et al. (2009) Microwave synthesis of fluorescent carbon nanoparticles with electrochemiluminescence properties. Chem Commun (34): 5118-5120.

6. Li H, Ming H, Liu Y, Yu H, He XD, et al. (2011) Fluorescent carbon nanoparticles: electrochemical synthesis and their $\mathrm{pH}$ sensitive photoluminescence properties. New J Chem 35(11): 2666-2670.

7. Khan S, Gupta A, Verma NC, Nandi CK (2015) TimeResolved Emission Reveals Ensemble of Emissive States as the Origin of Multicolor Fluorescence in Carbon Dots. Nano Lett 15(12): 8300-8305.

8. Das A, Gude V, Roy D, Chatterjee T, De CK, et al. (2017) On the Molecular Origin of Photoluminescence of Nonblinking Carbon Dot. J Phys Chem C 121(17): 9634-9641.

9. Sharma A, Gadly T, Gupta A, Ballal A, Ghosh SK, et al. (2016) Origin of Excitation Dependent Fluorescence in Carbon Nanodots. J Phys Chem Lett 7(18): 36953702.

10. Kalytchuk S, Polakova K, Wang Yu, Froning JP, Cepe K, et al. (2017) Carbon Dot Nanothermometry: Intracellular Photoluminescence Lifetime Thermal Sensing. ACS Nano 11(2): 1432-1442.

11. Sun X, Zhang Q, Yin K, Zhoua S, Li H (2016) Fluorescent vesicles formed by simple surfactants induced by oppositely-charged carbon quantum dots. Chem Commun 52(81): 12024-12027.

12. Nandi S, Malishev R, Bhunia SK, Kolusheva S, Jopp J, et al. (2016) Lipid-Bilayer Dynamics Probed by a Carbon Dot Phospholipid Conjugate. Biophysical Journal 110(9): 2016-2025.

13. Kong W, Liu J, Liu R, Li H, Liu Y, et al. (2014) Quantitative and real-time effects of carbon quantum dots on single living HeLa cell membrane permeability. Nanoscale 6(10): 5116-5120.

14. Zhu S, Song Y, Zhao X, Shao J, Zhang J, et al. (2015) The photoluminescence mechanism in carbon dots (graphene quantum dots, carbon nanodots, and polymer dots): Current state and future perspective. Nano Research 8(2): 355-381.

15. Tanford C (1980) The Hydrophobic Effect: Formation of Micelles and Biological Membranes. 2n (Edn.), Wiley, New York.

16. Rangel-Yagui CO, Tavares AP, Tavares LC (2005) Micellar solubilization of drugs. J Pharm Sci 8(2): 147165. 